\title{
Possible IgG4-related respiratory disease (IgG4-RRD) - a rare presentation as a Pancoast tumour-like
}

Inês S. Pereira 1, Diogo Fonseca 2, Margarida Dias 1, Ana Barroso 1, Patrícia Pinto 2, Daniela Machado 1

\author{
${ }^{1}$ Pulmonology Department, Centro Hospitalar de Vila Nova de Gaia/Espinho, Portugal. \\ ${ }^{2}$ Rheumatology Department, Centro Hospitalar de Vila Nova de Gaia/Espinho, Portugal. \\ *Corresponding author: I.S.Pereira. Centro Hospitalar de Vila Nova de Gaia/Espinho, Pulmonology Department. R. \\ Conceição Fernandes S/N 4434-502 Vila Nova de Gaia, Portugal. E-mail: ines.sucena@gmail.com
}

We declare that the patient approved the case report publishing by signing a written informed consent form. The case report followed the ethical guidelines established by the Declaration of Helsinki.

Received on: Sep 2, 2021. Accepted on: Sep 30, 2021. Available online: Oct 14, 2021.

\begin{abstract}
IgG4-related respiratory disease (IgG4-RRD) is a recently recognized disease that can have multiple presentations. In many cases, the exclusion of malignancy is essential. We present a case of a male patient with right omalgia and supraclavicular swelling. Radiologic exams showed a lung mass suggestive of a Pancoast tumour in the right upper lobe. Patient underwent transthoracic biopsies that revealed fibromuscular tissue with fibrosis, lymphoplasmacytic infiltrate and a significant increase in IgG4 cells with 15\% IgG4/IgG ratio. He started on prednisolone with clinical and radiologic improvement. As it is a heterogeneous disease it is important that clinicians are aware of the possible presentations.
\end{abstract}

Keywords: Immunoglobulin G4-Related Disease; Pancoast; Lung mass.

\section{Introduction}

IgG4-RRD is a recently recognized immune-mediated and usually multiorgan disease that presents many times as a tumorous lesion [1-3]. It may affect the lungs in different forms but can emerge as a pulmonary pseudotumour $[2,4]$.
The exclusion of a neoplastic process is fundamental to the correct diagnosis of a IgG4-RRD [2]. To the best knowledge of the authors, the initial presentation similar to a Pancoast tumour has not yet been described. The purpose of this case report is to improve scientific knowledge about the possible presentations of this rare disease. 


\section{Case report}

A fifty-two years old, non-smoker and not exposed to second hand tobacco smoke, male patient with a personal history of gastritis medicated with Omeprazole, presented right omalgia and supraclavicular swelling.

An ultrasound of the right shoulder was performed, which revealed cuff tendinosis and a

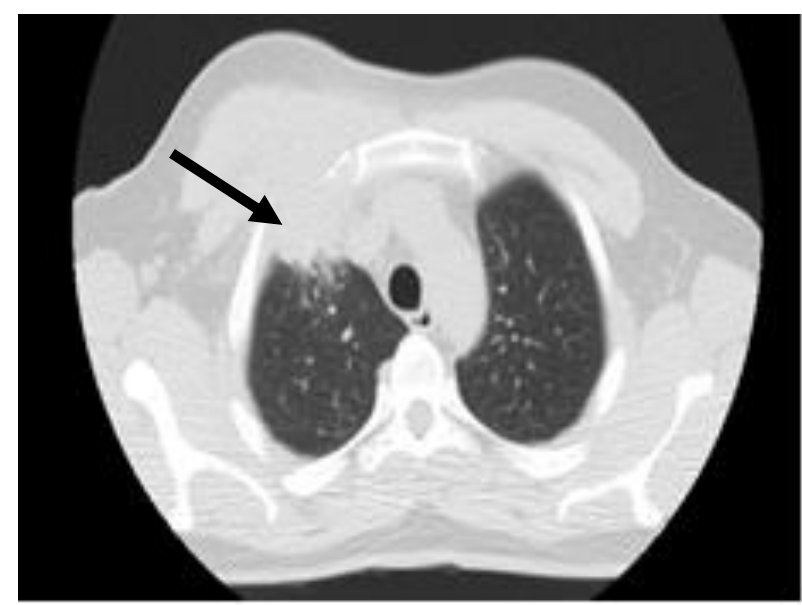

heterogeneous hypoechoic area posterior to the pectoralis major muscle.

He performed a chest Computed Tomography (CT) for further clarification which revealed a tumour mass suggestive of a Pancoast tumour in the right upper lobe measuring $46 \times 34 \mathrm{~mm}$. In Figure 1 we can see the lung mass on a CT scan performed just before the first transthoracic biopsy.

Figure 1. CT image (performed just before the first transthoracic biopsy) of lung mass suggestive of Pancoast tumour (arrow) in pulmonary and mediastinal window.

An 18-fluorodeoxyglucose positron emission tomography (18-FDG PET) was performed, showing high 18FDG uptake (maximum SUV 16.6) in the identified mass extending to the soft tissues of the chest wall and to the right hilar and right paratracheal ganglia.

Due to high suspicion of lung cancer presenting as a Pancoast tumour, it was performed two transthoracic biopsies at different times that revealed fibromuscular tissue with fibrosis,

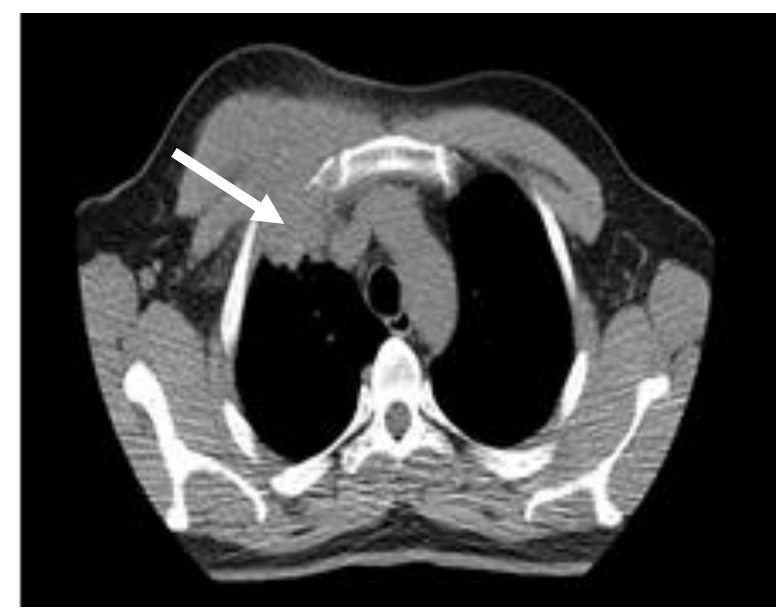


Immunological study revealed increased sedimentation velocity $(62 \mathrm{~mm} / \mathrm{h})$, slightly increased Immunoglobulin G (1490mg/dl) with increased Immunoglobulin G4 $(247 \mathrm{mg} / \mathrm{dl})$ which established an IgG4/IgG serum ratio of $16.6 \%$.

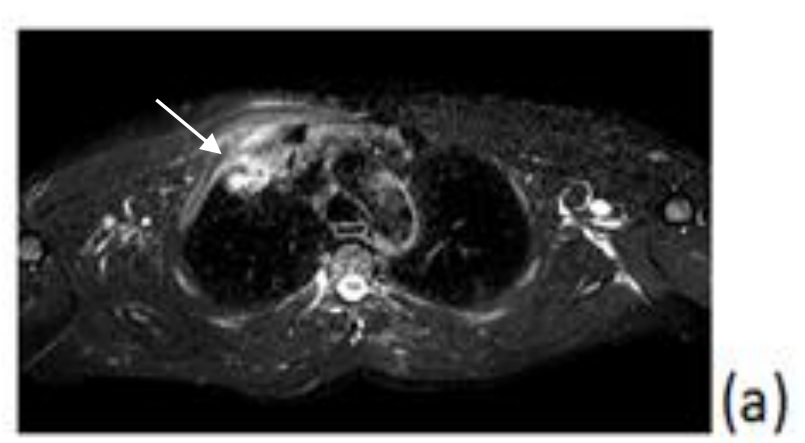

Patient was referred to Rheumatology, and initiated prednisolone $40 \quad \mathrm{mg} /$ day $\quad$ (0.5 $\mathrm{mg} / \mathrm{kg} /$ day) with clinical improvement, decrease in the size of the lesion (Figure 2) and decrease in IgG4 serologic values.

Figure 2. MRI identifying lung mass (arrow) before (a) and after (b) treatment

There was a relapse with worsening of the pain and swelling with the tapering of the Prednisolone dose. After relapse it was performed a third biopsy to confirm the diagnosis that revealed muscular tissue with sclerosing areas and inflammatory infiltrate with > 10 IgG4 cells/HPF, which was also suggestive of IgG4-RRD. Corticosteroid therapy was increased.

After that, patient was hospitalized because of a bacteraemia due to $S$. aureus methicillin sensitive, probably in the context of systemic corticosteroid therapy and biopsy puncture. This hospitalization was complicated with a Stevens-Johnson syndrome, which resolved after receiving immunoglobulins and corticosteroids. After hospitalization,

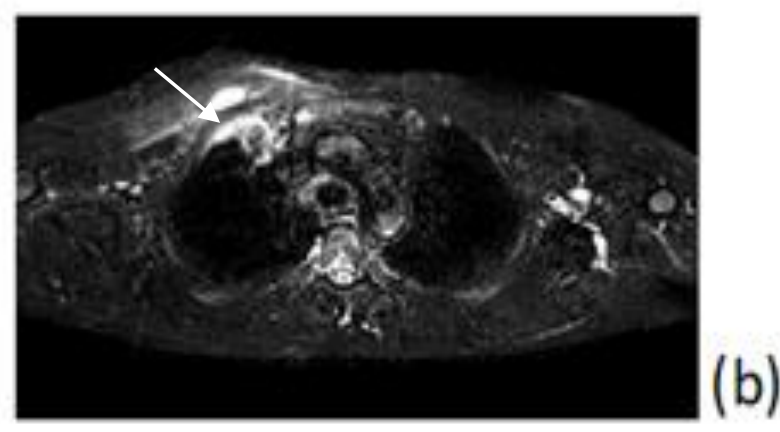

(b) 
The increase in serum IgG4 is not diagnostic but is suggestive of this disease, usually with IgG4 > $135 \mathrm{mg} / \mathrm{dl}$ [4-5] and serum IgG4/IgG ratio >10 [2], which was found in our patient. The histopathological features of IgG4-RRD are a lymphoplasmacytic infiltrate, fibrosis and obliterative phlebitis [1-2, 4]. Most authors defend the presence of $>10$ IgG4 /HPF and an IgG4/IgG ratio > $40 \%$ in tissue sample to make the diagnosis $[2,4,5]$.

In our case, the patient had $>10$ IgG4/HPF but not a ratio $>40 \%$. However, the clinical presentation suggestive of IgG4-RRD, the increase in serum IgG4, the absence of another diagnosis to explain the changes found, and the clinical and imaging initial response to corticosteroid therapy allowed us to make a presumptive diagnosis of this pathology, despite not meeting all the criteria.

The treatment of IgG4-RRD is based on corticosteroid therapy with good initial response but with a relapse of $40-76 \%$ of patients, usually during tapering of the corticosteroid dose [2-3]. The use of glucocorticoid sparing therapies is still being studied [2-3]. Due to lack of long-term studies of these patients, long-term prognosis cannot be established. However, it is known that, without treatment, this disease can be progressive and cause considerable morbidity and mortality [2].
In conclusion, IgG4-RRD is a recently identified disease whose diagnosis is often difficult with a highly variable clinical presentation. When this disease presents as a pulmonary nodule or mass, the exclusion of cancer is fundamental [2-3]. Improving knowledge about its possible presentations is essential for clinicians to be alert and suspicious of this condition.

\section{References}

[1] Wallace ZS, Naden RP, Chari S, Choi H, Della-Torre E, Dicaire JF, Hart PA, Inoue D, Kawano M, Khosroshahi A, Kubota K, Lanzillotta M, Okazaki K, Perugino CA, Sharma A, Saeki T, Sekiguchi H, Schleinitz N, Stone JR, Takahashi N, Umehara H, Webster G, Zen Y, Stone JH; American College of Rheumatology/European League Against Rheumatism IgG4-Related Disease Classification Criteria Working Group. The 2019 American College of Rheumatology/European League Against Rheumatism Classification Criteria for IgG4-Related Disease. Arthritis Rheumatol. 2020 Jan;72(1):7-19. doi: 10.1002/art.41120.

[2] Abraham M, Khosroshahi A. Diagnostic and treatment workup for IgG4-related disease. Expert Rev Clin Immunol. 2017 Sep;13(9):867-875. doi: 10.1080/1744666X.2017.1354698.

[3] Khosroshahi A, Wallace ZS, Crowe JL, Akamizu T, Azumi A, Carruthers 
MN, Chari ST, Della-Torre E, Frulloni L, Goto H, Hart PA, Kamisawa T, Kawa S, Kawano M, Kim MH, Kodama Y, Kubota K, Lerch MM, Löhr M, Masaki Y, Matsui S, Mimori T, Nakamura S, Nakazawa T, Ohara H, Okazaki K, Ryu JH, Saeki T, Schleinitz N, Shimatsu A, Shimosegawa T, Takahashi $\mathrm{H}$, Takahira M, Tanaka A, Topazian M, Umehara H, Webster GJ, Witzig TE, Yamamoto M, Zhang W, Chiba T, Stone JH; Second International Symposium on IgG4Related Disease. International Consensus Guidance Statement on the Management and Treatment of IgG4Related Disease. Arthritis Rheumatol. 2015 Jul;67(7):1688-99. doi: 10.1002/art.39132.

[4] Umehara H, Okazaki K, Nakamura T, Satoh-Nakamura T, Nakajima A, Kawano M, Mimori T, Chiba T. Current approach to the diagnosis of IgG4related disease - Combination of comprehensive diagnostic and organspecific criteria. Mod Rheumatol. 2017 May;27(3):381-391. doi: 10.1080/14397595.2017.1290911.

[5] Matsui S, Yamamoto H, Minamoto S, Waseda Y, Mishima M, Kubo K. Proposed diagnostic criteria for IgG4related respiratory disease. Respir Investig. 2016 Mar;54(2):130-2. doi: 10.1016/j.resinv.2015.09.002.

Conflict of interest: The author declares no conflicts of interest.
Acknowledgements: None.

Funding: None.

How to cite this article: Pereira IS, Fonseca D, Dias M, Barroso A, Pinto P, Machado D. Possible IgG4-related respiratory disease (IgG4-RRD) - a rare presentation as a Pancoast tumour-like. Brazilian Journal of Case Reports. 2021 Jul-Sep;01(4):45-49. 